\title{
STATISTICAL TEXTURE CLASSIFCATION VIA HISTOGRAMS OF WAVELET FILTERED IMAGES
}

\author{
ALAN WEE-CHUNG LIEW ${ }^{1}$, JUN JO ${ }^{1}$, \\ TAE BYONG CHAE ${ }^{2}$ and YONG-SIK CHUN ${ }^{2}$ \\ ${ }^{1}$ School of Information and Communication Technology, \\ Gold Coast campus, Griffith University, QLD4222, Australia \\ \{a.liew@griffith.edu.au, j.jo@griffith.edu.au\} \\ ${ }^{2}$ Department of KOMPSAT-5 System Engineering \& Integration \\ Korea Aerospace Research Institute \\ POB 113 Yusung Daejeon, 305-333, KOREA \\ \{sik@kari.re.kr\}
}

\begin{abstract}
:
We show how the first order statistic, i.e. the histogram, of the wavelet filtered image is related to the higher order statistic of the original image. We then propose a texture classification method that uses the histogram information of the filtered images as the feature vector. Classification experiments show that the proposed method achieves better classification result than the Gabor wavelet filtering method using only the mean and standard deviation of the absolute filtered images as feature vectors.
\end{abstract}

\section{Keywords:}

Texture classification; wavelet filter; spatial grey level dependence

\section{Introduction}

Texture perception is an important part of vision since objects can often be distinguished by their characteristic textures despite their similarity in colors or shapes. With the advent of digital storage media, the use of textural information in performing content-based image retrieval from digital image library has become indispensable.

Most texture analysis methods can be categorized into either statistical approach or structural approach. The statistical approach attempts to use the statistical properties of the spatial distribution of grey level as texture descriptors. The structural approach, on the other hand, conceives of texture as an arrangement of a set of spatial sub-patterns or texture primitives according to some placement rules. Recently, wavelet filtering-based methods are being proposed for texture analysis and classification. In this paper, we discuss two well-known statistical texture classification methods, called the spatial grey level dependence (SGLD) method [1] and the wavelet filtering method [2, 6] and point out the underlying relationship between these two methods. We then propose a new classification method that combine features from these two methods and give experimental results to illustrate its performance.

\section{Texture Classification Methods}

The SGLD method is based on the estimation of the second-order joint conditional probability distribution $p(i, j \mid d, \theta)$ where each $p(i, j \mid d, \theta)$ is the probability of going from the grey level $i$ to the grey level $j$ given the intersample distance $d$ and the direction in angle $\theta$. This method was inspired by the extensive psychovisual texture discrimination studies which showed human are sensitive to the second-order statistical variation in texture [5]. To characterize a texture adequately, a set of inter-sample distances and directions is generally used [1].

If the grey level range is quantized into $N$ levels, then each $\left(d_{k}, \theta_{k}\right)$ will produce an $\mathrm{N} \times \mathrm{N}$ co-occurrence matrix. Instead of using these matrices directly, a number of parameters, e.g., angular second moment, contrast and entropy, are usually computed from each of these matrices and are used as texture descriptors. However, this inevitably reduces the discriminative power of the SGLD method. In addition, these parameters are usually proposed in an ad hoc manner and may be suboptimal.

In the wavelet filtering method, each wavelet filter at a particular orientation produces an output image with localized spectrum at different location in the frequency plane. The localized spatial-frequency resolution of the wavelet function allows a local analysis of the image at different frequency resolution. The mean $\mu$ and standard deviation $\sigma$ of the absolute image are usually calculated from each of the output images and are used as texture 
descriptors ([2] uses both $\mu$ and $\sigma$ where as [6] uses only $\mu$ )

Since many image structures, i.e., directionality, periodicity, and coarseness, and their properties can be inferred from the local spectrum, the wavelet filtering method can produce meaningful texture descriptors describing these image structures. However, in attempting to characterize the local spectrum using only two parameters $\mu$ and $\sigma$, much useful information are lost. Moreover, unlike the SGLD method, this method does not appear to relate to the extensive psychovisual texture discrimination studies.

\section{First Order Statistics of the Filtered Image}

We focus our attention on the 1-D case for clarity of presentation although the analysis extends directly to 2-D. Let $\left\{h_{n}\right\}_{n=0, \ldots, N-1}$ be the coefficients of an N-tap filter. For a discrete 1-D signal $f$ of length $L$, the convolution of the filter and the signal can be written as

$$
y_{t}=\sum_{n=0}^{N-1} h_{n} f_{t+n}, t=0, \ldots, L-1
$$

where we assume that the signal has been reflected about the origin. Let $F$ and $Y$ be the random variables associated with the input image and the filter output respectively. Clearly, the range of value for $Y$ is dependent on the range of grey level value $R(F)$ of the input signal and the filter coefficients. The probability that $Y$ assumes a particular value, say, $y_{i}$, is then given by

$$
\begin{aligned}
& p\left(Y=y_{i}\right)=p\left(\sum_{n=0}^{N-1} h_{n} f_{n}=y_{i}\right) \\
&=\sum_{f_{0}} \ldots \sum_{f_{N-2}} p\left(f_{0}, f_{1}, \ldots, f_{N-2}, \frac{1}{h_{N-1}}\left(y_{i}-h_{0} f_{0}\right.\right. \\
&\left.\left.-h_{1} f_{1}-\cdots-h_{N-2} f_{N-2}\right)\right)
\end{aligned}
$$

where $\left\{f_{0}, f_{1}, \ldots, f_{N-2}\right\} \in R(F)$. In terms of the N-order joint conditional probability,

$$
\begin{gathered}
p\left(Y=y_{i}\right)=\sum_{f_{0}} \ldots \sum_{f_{N-2}} p\left(f_{0}, f_{1}, \ldots, f_{N-2}, \frac{1}{h_{N-1}}\left(y_{i}-h_{0} f_{0}\right.\right. \\
\left.-h_{1} f_{1}-\cdots-h_{N-2} f_{N-2}\right) \mid d_{1}=d_{2}=\cdots \\
\left.=d_{N-1}=1\right)
\end{gathered}
$$

where the inter-sample distance $d_{k}$ denotes the distance between sample $k-1$ and sample $k$. Note that the subscript of $f$ in (2) and (3) is not a time index but rather denotes the relative position of the elements in the signal vector $f$ with respect to the filter coefficients. For $N=2$, (3) becomes

$$
p\left(Y=y_{i}\right)=\sum_{f_{0}} p\left(f_{0}, \frac{1}{h_{1}}\left(y_{i}-h_{0} f_{0}\right) \mid d_{1}=1\right)
$$

Let $M$ be the co-occurrence matrix with elements $p\left(f_{0}, f_{1} \mid d_{1}=1\right)$ and with the $\mathrm{x}$ and $\mathrm{y}$-axis labeled by the random variables $F_{0}$ and $F_{1}$ respectively. Then (4) implies that the probability of the filter output $Y=y_{\mathrm{i}}$ is given by the sum of the elements of the co-occurrence matrix $M$ lying on the straight line with gradient given by $h_{0} / h_{1}$,

$$
F_{1}=\frac{y_{i}}{h_{1}}-\frac{h_{0}}{h_{1}} F_{0}
$$

Similarly, for $N=3$, the probability of $Y=y_{i}$ is given by the sum of the elements of a $3-D$ joint conditional probability matrix lying on the plane

$$
F_{2}=\frac{y_{i}}{h_{2}}-\frac{h_{0}}{h_{2}} F_{0}-\frac{h_{1}}{h_{2}} F_{1}
$$

Thus, the first-order statistics, i.e., the histogram, of a signal filtered by a 2-tap filter provides a description (i.e. histogram of the sum of elements of $M$ at parallel lines with gradient $h_{0} / h_{1}$ ) of the second-order statistics, i.e., the cooccurrence matrix, of the original signal. In general, given an N-tap filter with $N$ non-zero filter coefficients, the firstorder statistics of the filtered signal provides a description of the N-order statistics of the original signal. The filtering operation therefore produces a statistical simplification of the original signal. It should be noted that while the firstorder statistics of the filtered signal can be obtained from the higher order statistics of the original signal, the converse is not true.

The above analysis shows how the filter outputs are related to the higher order statistics of the original image. So rather than trying to summarize the N-order statistics of the original image with simple statistics such as $\mu$ and $\sigma$, the complete histogram of the filtered image would provide better discriminating power. Although the above discussion is applicable to any filtering scheme, the wavelet filtering scheme does have some advantages. Firstly, the localized spatial-frequency support of the wavelet functions enables local analysis of texture elements. Secondly, the wavelet multiresolution framework allows texture to be analyzed at different resolution in a consistent manner. 


\section{Proposed Classification Method and Results}

In the proposed method, a texture image is first filtered with a set of cortex wavelet filters [4] tuned to 4 orientations and 4 scales. A schematic of the spatial frequency plane division of this filter is shown in Fig. 1.

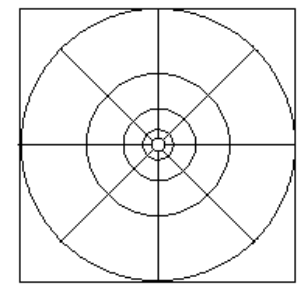

Fig. 1: Spatial frequency plane division of the cortex wavelet filters. The scales are in octave, orientation in $45^{\circ}$.

After filtering, the grey level cumulative density function (cdf) of each filtered image is computed and the grey levels corresponding to the $0.1 k \quad(k=1$ to 10$)$ probability of the cdf are used as the texture features. The features from the 16 filtered images, together with the features from the original image, the low-pass and highpass residues which are obtained similarly, are concatenated to form the raw feature vector for the texture image.

We performed a classification experiment in which the task is to assign a test image to one of the known texture classes. We collected $35512 \times 512$ images consisting of 33 classes from the Brodatz texture set [5]. Images of size $128 \times 128$ are extracted from these larger images and randomly rotated, and 10 images from each class are used for training while 30 images from each class are used for testing. Some of the test images are shown in Fig. 2.
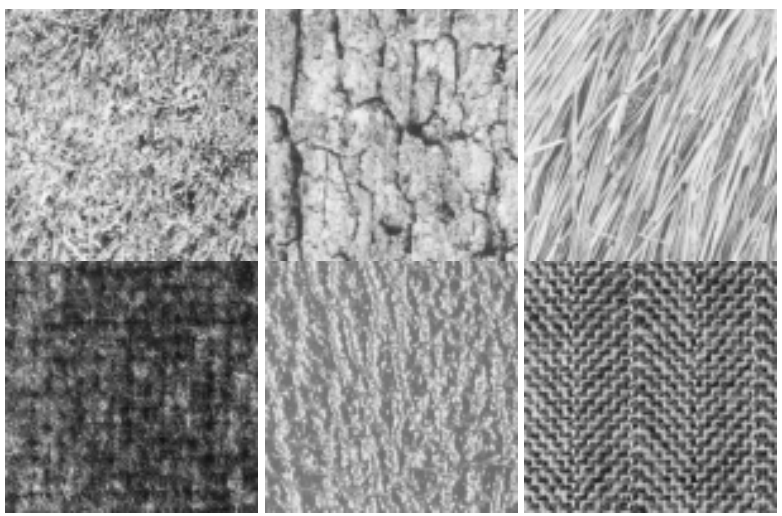

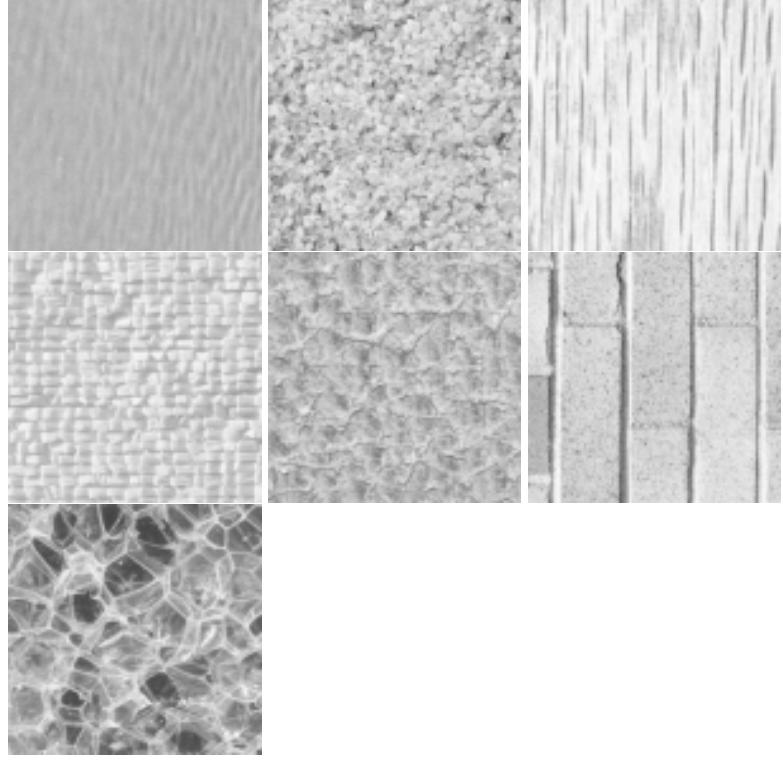

Fig. 2: Some test images obtained from [7]. Left to right, top to bottom: Grass, Bark, Straw, Woolen cloth, Herringbone weave,

Pressed calf leather, Beach sand, Water, Wood grain, Raffia, Pigskin, Brick wall, Plastic bubbles.

For the training phase, the raw feature vector for each training image is first computed. The raw feature vectors then undergo Principle Component Analysis (PCA) followed by Linear Discriminant Analysis (LDA) [3]. During PCA, only the 80 most significant elements of the feature vector corresponding to the 80 largest eigenvalues are kept. At the LDA stage, the length of the feature vector is further reduced to 10 . Finally, the class centroids are computed from the feature vectors.

During testing, the feature vector of the test image is projected onto the PCA and LDA spaces using the projection matrices obtained at the training phase. The resulting feature vector $x$ is then compared to the class centroid $c_{i}$ using the following distance metric,

$$
d\left(x, c_{i}\right)=\sum_{j=1}^{10}\left(\frac{x(j)-c_{i}(j)}{\sigma\left(c_{i}(j)\right)}\right)^{2}
$$

where $\sigma\left(c_{i}(j)\right)$ is the standard deviation of the $j$ th element in the class centroid $c_{i}$ of class $i$. For comparison, we also performed the classification experiment using the Gabor wavelet filtering method as in [2]. Let the Gabor wavelet transform of an image $I(x, y)$ be given by

$$
W_{m n}(x, y)=\int I\left(x_{l}, y_{l}\right) g_{m n}\left(x-x_{l}, y-y_{l}\right) d x_{l} d y_{l}
$$

where $g_{m n}$ is the Gabor wavelet at scale $m$ and orientation 
$n$. The $\mu_{m n}$ and $\sigma_{m n}$ is given by

$$
\begin{gathered}
\mu_{m n}=\iint\left|W_{m n}(x, y)\right| d x d y \\
\sigma_{m n}=\sqrt{\iint\left(\left|W_{m n}(x, y)\right|-\mu_{m, n}\right)^{2} d x d y}
\end{gathered}
$$

In [2], $m$ and $n$ are chosen to be 4 and 6, respectively, giving a total of 24 filtered images. This gives a feature vector of length 48. To allow a direct comparison, the Gabor texture feature vectors also undergo PCA and LDA to give a final length of 10 and use the same distance metric as in (7). Table 1 presents the average classification performance of each class for both methods. The overall average classification performance of our approach and Gabor approach are $98.4 \%$ with standard deviation of 3.9 and $92.8 \%$ with standard deviation of 9.6 respectively. Thus, with a smaller standard deviation, our approach is more robust and reliable.

\section{Conclusions}

We presented the theoretical basis for using the histograms of the wavelet filtered images as texture descriptor and showed its relationship to the spatial grey level dependence matrix when the number of filter tap equals to 2. Experimental results and comparison with the well known wavelet based texture classification method of Manjunath [2] have shown the feasibility and robustness of our approach. The histogram of the wavelet filtered images is able to provide rich description that better characterizes the texture than the simple mean and standard deviation of the absolute image. The method developed can be used to perform image region segmentation.

\section{Acknowledgment}

This work is supported by Korean Aerospace Research Institute.

\section{References}

[1] R.M. Haralick, K. Shanmugam, and I. Dinstein, "Textural features for image classification", IEEE Trans. Systems Man and Cybernetics, Vol. 3, No. 6, pp. 610-621, Nov. 1973.

[2] B.S. Manjunath, and W.Y. Ma, "Texture features for browsing and retrieval of image data”, IEEE Trans. Pattern Analysis and Machine Intelligent, Vol. 18, No. 8, pp. 837-842, Aug 1996.
[3] D.L. Swets, and J. Weng, "Using discriminant eigenfeatures for image retrieval”, IEEE Trans. Pattern Analysis and Machine Intelligent, Vol. 18, No. 8, pp. 831-836, Aug 1996

[4] A.B. Watson, "The cortex transform: Rapid computation of simulated neural images”, Computer Vision, Graphics, and Image Processing, Vol. 39, pp. 311-327, 1987.

[5] P. Brodatz, "Textures: A Photographic Album for Artists and Designers”, New York: Dover, New York, 1966.

[6] T. Chang and C.-C.J. Kuo, "Texture Analysis and Classification with Tree-Structured Wavelet Transform”, IEEE Trans. Image Processing, vol. 2, no. 4, pp. 429-441, Oct. 1993.

[7] http://sipi.usc.edu/database/database.cgi?volume=text $\underline{\text { ures }}$

\begin{tabular}{|c|c|c|}
\hline Class & Our method & Gabor filtering \\
\hline 1 & 100 & 93.3 \\
\hline 2 & 100 & 100 \\
\hline 3 & 100 & 100 \\
\hline 4 & 100 & 100 \\
\hline 5 & 100 & 51.7 \\
\hline 6 & 96.7 & 86.7 \\
\hline 7 & 100 & 100 \\
\hline 8 & 100 & 96.7 \\
\hline 9 & 100 & 96.7 \\
\hline 10 & 100 & 100 \\
\hline 11 & 100 & 93.3 \\
\hline 12 & 100 & 100 \\
\hline 13 & 100 & 96.7 \\
\hline 14 & 100 & 100 \\
\hline 15 & 96.7 & 86.7 \\
\hline 16 & 93.3 & 100 \\
\hline 17 & 100 & 86.7 \\
\hline 18 & 96.7 & 96.7 \\
\hline 19 & 100 & 100 \\
\hline 20 & 96.7 & 76.7 \\
\hline 21 & 100 & 90 \\
\hline 22 & 100 & 90 \\
\hline 23 & 100 & 100 \\
\hline 24 & 100 & 100 \\
\hline 25 & 93.3 & 93.3 \\
\hline 26 & 80 & 83.3 \\
\hline 27 & 100 & 100 \\
\hline 28 & 100 & 96.7 \\
\hline 29 & 100 & 93.3 \\
\hline 30 & 100 & 100 \\
\hline 31 & 100 & 100 \\
\hline 32 & 93.3 & 100 \\
\hline 33 & 100 & 86.7 \\
\hline Average & $98.4(\mathrm{~s} . \mathrm{d}=3.9)$ & 92.8 (s.d=9.6) \\
\hline
\end{tabular}

TABLE 1. AVERAgE CLASSIFICATION PERFORMANCE (\%) 